\title{
The study of Psychiatric Disorders in patients with Thyroid Disorder at the tertiary care centre in Western Region of Nepal
}

\author{
Thapa DK' ${ }^{1}$ padhyaya TL21amichhaneN ${ }^{3}$ Subedi $\mathrm{S}^{4}$
}

1. Assistant Professor, Department of Psychiatry, GMC, Pokhara, Nepal, 2. Assistant Professor, Internal Medicine, GMC, Pokhara, Nepal, 3. Associate Professor, Department of Psychiatry, GMC, Pokhara, Nepal 4. Assistant Professor, Department of Psychiatry, UCMS, Bhairahawa, Nepal

Email Corresponding Author: ddthapa@hotmail.com

\section{Abstract}

Background:Recent advances in biotechnology have led to an improved understanding of the impact of thyroid functions on the adult, mature brain. This development has been particularly helpful in elucidating the role of thyroid hormones in the pathophysiology of psychiatric disorders. The aim of the study was to evaluate the occurrence of various psychiatric disorders in adult patients affected by different thyroid disorders.

Methods and materials:This was a descriptive, cross- sectional, hospital based and a collaborative study between the internal medicine and the psychiatry department at Gandaki Medical College, Pokhara, Nepal. Based on the ICD- 10 psychiatric diagnosis, 60 consecutive consenting subjects of any gender above 18 years of age were included in the study. The duration of the study was 6 months.

Results:The total numbers of subjects enrolled in the study were 60. The female consisted of $81.7 \%$ (49). The mean age was $39.62 \pm 12.85$ years. Regarding the thyroid disorder; $76.7 \%$ (46) had hypothyroidism, 15\% (9) had sub clinical hypothyroidism, and both the hyperthyroidism and Hashimoto thyroiditis were found in $3.3 \%$ (2) of the cases while papillary carcinoma was found in $1.7 \%$ (1) case. Determination of psychiatric co-morbidity showed that; greater numbers of the subjects i.e. $48.3 \%$ (29) had neurotic, stress- related and somatoform disorders (F40-F48), 26.7\% (16) had mood (affective) disorder (F30- F39), 13.3\% (8) had simultaneously two psychiatric disorder, 10\% (6) had headache and only $1.7 \%$ (1) had lack or loss of sexual desire (F52.0).

Conclusion: Compared to males, Females are more likely to have thyroid disorder. Clinician has to be alert to the possible underlying psychiatric disorder in subjects with any thyroid disorder.

Keywords: Female, Thyroid disorder, Psychiatric disorder

\section{INTRODUCTION}

Thyroid gland is essential for normal human development and maintenance. ${ }^{1}$ Recent studies suggest that thyroid hormones have direct and important effect on mature brain function. ${ }^{2}$ During brain development, iodine deficiency, maternal thyroid dysfunction, and neonatal thyroid malformations together with genetic factors may cause permanent neurological deficits with severe mental retardation and cretinism. ${ }^{3}$
For more than a century, science has recognized the connection between the thyroid axis and several commonly experienced psychiatric disorders. Small changes in thyroid hormone levels, within the normal range, may have significant effects on cerebral thyroid function. This may manifest as alterations in mood, behavior and cognition. ${ }^{2}$ 
Unlike in children, in adults, the effects of thyroid hormones on mental functioning are said to be less striking. Most psychiatric patients may not have overt thyroid dysfunction. If a thyroid abnormality is present, it is usually mild and may be considered as a risk factor for mental disorder rather than as a causal factor. ${ }^{3}$

But the recent advances in biotechnology have led to an improved understanding of the impact of thyroid functions on the adult, mature brain. This development has been particularly helpful in elucidating the role of thyroid hormones in the pathophysiology of psychiatric disorders. ${ }^{4}$

Now the several studies have underlined the high prevalence of psychiatric symptoms and disorders in thyroid diseases. 5

Slowly progressive changes in thyroid hormone levels are more likely to be associated with a picture of chronic anxiety, increased fatigability and psychomotor slowing. The severity of mental symptoms is greater in elderly patients and, as noted, in patients with rapidly changing thyroid hormone levels. 6

Psychiatric disorders, loss of consciousness, movement disorders and weakness may all be manifestations of primary thyroid disease. ${ }^{1} \mathrm{In}$ adult patients with frank thyroid disease, mental symptoms are usually limited to depression, anxiety, or reversible cognitive decline. ${ }^{3}$ Rapid cycling and other refractory forms of bipolar disorder have been particularly highlighted, since the prevalence of thyroid dysfunction appears to be greater in such forms of the disorder. ${ }^{4}$ Some patients may not meet the full criteria of a specific mental disorder, but they will not be free of mental symptoms such as tension, hyperactivity, or autonomic symptoms. ${ }^{3}$

\section{METHOD}

This was a descriptive, cross- sectional, hospital based and a collaborative study between the internal medicine and the psychiatry department at Gandaki Medical College, Pokhara, Nepal. The aim of this study was to evaluate the occurrence of psychiatric disorders in adult patients affected by different thyroid diseases. The patients diagnosed with any thyroid disorder, who were suspected to have suffered from psychiatric ailments, were referred by the physician to psychiatry outpatient department. Those patients were thoroughly interviewed and examined by the psychiatrists and the psychiatric diagnoses were based on the ICD-10. The results were then interpreted. The duration of the study was 6 months, from March to August 2013. The consecutive consenting subjects of any gender above 18 years of age were included in the study. Patients with other multiple medical comorbidities were excluded from the study.

\section{RESULTS}

The total numbers of subjects enrolled in the study were 60 . Female consisted of $81.7 \%$ (49) while $18.3 \%$ (11) were the males. The mean age was $39.62 \pm 12.85$ years while the age ranged from 20 years to 70 years. The $86.7 \%$ (52) of the subjects were married while $13.3 \%$ (8) were single. Regarding the thyroid disorder; $76.7 \%$ (46) had hypothyroidism, $15 \%$ (9) had sub clinical hypothyroidism, both the hyperthyroidism and Hashimoto thyroiditis were found in 3.3\% (2) of the cases while papillary carcinoma was found in 1.7\% (1) patient(See Table 1)

Table 1: Showing the distribution of thyroid disorder $(n=60)$ :

\begin{tabular}{|c|c|c|}
\hline Thyroid disorder & $\begin{array}{c}\text { Frequency } \\
\text { (n=60) }\end{array}$ & $\begin{array}{c}\text { Percentage } \\
(\mathbf{\%})\end{array}$ \\
\hline Hypothyroidism & 46 & 76.7 \\
\hline $\begin{array}{c}\text { Subclinical } \\
\text { Hypothyroidism }\end{array}$ & 9 & 15 \\
\hline Hyperthyroidism & 2 & 3.3 \\
\hline $\begin{array}{c}\text { Hashimoto } \\
\text { thyroiditis }\end{array}$ & 2 & 3.3 \\
\hline Papillary Carcinoma & 1 & 1.7 \\
\hline Total & 60 & 100 \\
\hline
\end{tabular}

Determination of psychiatric co-morbidity showed that; greater numbers of the subjects i.e. $48.3 \%$ (29) had neurotic, stress- related and somatoform disorders (F40- F48), 26.7\% (16) had mood (affective) disorder (F30- F39), 13.3\% (8) had simultaneously two psychiatric disorders, $10 \%$ (6) had headache and only $1.7 \%$ (1) had lack or loss of sexual desire (F52.0). (See Table 2) 
Table 2: Showing the distribution of psychiatric comorbidity in subjects with thyroid disorder $(n=60)$ :

\begin{tabular}{|c|c|c|c|}
\hline $\begin{array}{l}\text { Category of psychiatric } \\
\text { comorbidity }\end{array}$ & Types of psychiatric disorder & $\begin{array}{l}\text { Frequency } \\
(n=60)\end{array}$ & $\begin{array}{l}\text { Percentage } \\
(\%)\end{array}$ \\
\hline \multirow{8}{*}{$\begin{array}{l}\text { Neurotic, stress- related } \\
\text { and somatoform } \\
\text { disorders (F40- F48) }\end{array}$} & Anxiety disorder unspecified & 17 & \\
\hline & Somatization disorder & 5 & \\
\hline & Mixed anxiety depressive disorder & 2 & \\
\hline & Panic disorder & 2 & \\
\hline & Obsessive compulsive disorder & 1 & \\
\hline & Persistent somatoform pain disorder & 1 & \\
\hline & Neuroasthenia & 1 & \\
\hline & Total & 29 & 48.3 \\
\hline \multirow{6}{*}{$\begin{array}{l}\text { Mood (affective) disorder } \\
\text { (F30- F39) }\end{array}$} & Recurrent depressive disorder & 6 & \\
\hline & Bipolar affective disorder & 4 & \\
\hline & Depressive episode & 3 & \\
\hline & Dysthymia & 2 & \\
\hline & Mania & 1 & \\
\hline & $\begin{array}{c}\text { Total } \\
\end{array}$ & 16 & 26.7 \\
\hline \multirow{6}{*}{$\begin{array}{l}\text { Simultaneously occurring } \\
\text { psychiatric disorder }\end{array}$} & $\begin{array}{c}\text { Migraine headache with Somatization } \\
\text { disorder }\end{array}$ & 2 & \\
\hline & $\begin{array}{l}\text { Bipolar affective disorder with Harmful use } \\
\text { of alcohol }\end{array}$ & 2 & \\
\hline & $\begin{array}{l}\text { Migraine headache with Anxiety disorder } \\
\text { unspecified }\end{array}$ & 2 & \\
\hline & $\begin{array}{l}\text { Migraine headache with Depressive } \\
\text { episode }\end{array}$ & 1 & \\
\hline & $\begin{array}{c}\text { Migraine headache with Recurrent } \\
\text { depressive disorder }\end{array}$ & 1 & \\
\hline & Total & 8 & 13.3 \\
\hline \multirow{3}{*}{ Headache cases } & Migraine Headache & 5 & \\
\hline & Mixed Headache & 1 & \\
\hline & Total & 6 & 10 \\
\hline \multirow[t]{2}{*}{ F52.0 } & Lack or loss of sexual desire & 1 & 1.7 \\
\hline & Overall total & 60 & 100 \\
\hline
\end{tabular}

Among $48.3 \%$ (29) cases of neurotic, stressrelated and somatoform disorders i.e. (F40- F48) category, the distributions of disorders were; 17 cases of anxiety disorder unspecified, 5 cases of somatization disorder, 2 cases of mixed anxiety disorder and panic disorder each and a single case of obsessive- compulsive disorder, persistent somatoform pain disorder and neurasthenia.

Among $26.7 \%$ (16) cases of mood (affective) disorder i.e. (F30 - F39) category, the distribution of disorders were; 6 cases of recurrent depressive disorder, 4 cases of bipolar affective disorder, 3 cases of depressive episode, 2 cases of dysthymia and a single case of mania.

Among $13.3 \%$ (8) cases of simultaneously occurring psychiatric disorder, the distribution of disorders were; 2 cases of migraine headache with somatization disorder, 2 cases of bipolar affective disorder with harmful use of alcohol, 2 cases of migraine headache with anxiety disorder unspecified, a single case of migraine headache with depressive episode and a single case of migraine headache with recurrent depressive disorder. The diagnosis of headache was based on criteria of set forth by International Headache Society (IHS).

Among 10\% (6) cases of headache, 5 cases were of migraine headache and the remaining single case was of mixed headache.

Out of the 60 subjects, medical co- morbidity was found in $10 \%$ (6) of the cases only. Those being, 2 cases of iron deficiency anemia, and each case of 
ischemic heart disease, irritable bowel syndrome, diabetes mellitus and carpel tunnel syndrome.

Evaluation of the family history of thyroid disorder revealed that, majority of the subjects i.e. $93.3 \%$ (56) had no family history. In remaining $6.7 \%$ (4) of the cases, there was a positive history of hypothyroidism. (See Table 3)

Table 3: Showing the family history of thyroid and mental disorder:

\begin{tabular}{|c|c|}
\hline \multicolumn{2}{|c|}{ Family history of thyroid disorder } \\
\hline Positive & Hypothyroidism: 4 (6.7\%) \\
\hline Negative & $56(93.3 \%)$ \\
\hline Total & $60(100 \%)$ \\
\hline \multicolumn{2}{|c|}{ Family history of mental disorder } \\
\hline \multirow{2}{*}{ Positive } & Anxiety disorder unspecified: $3(5 \%)$ \\
\cline { 2 - 2 } & Bipolar affective disorder: 3 (5\%) \\
\cline { 2 - 2 } & Depression: $2(3.3 \%)$ \\
\cline { 2 - 2 } Negative & Migraine headache: $2(3.3 \%)$ \\
\hline Total & $50(83.3 \%)$ \\
\hline
\end{tabular}

About 83.3\% (50) did not have family history of mental disorder. Among the detected cases, anxiety disorder unspecified and bipolar affective disorder were found both in 5\% (3) of the cases while depression and migraine headache were found both in $3.3 \%$ (2) of the cases.

\section{DISCUSSION}

Thyroid disorder can present with variety of symptoms including the psychiatric symptoms. The current study was to determine the distribution of various thyroid disorder and the presenting comorbid psychiatric conditions among the patients attending Gandaki Medical College, a tertiary care centre at Pokhara, Nepal.

As shown in other studies, thyroid disorder is more common in female gender, and the rate rises with age, specially reaching $20 \%$ in women over 65.7 Among the thyroid disorder our study shows that; hypothyroidism was the most common occurrence $(76.7 \%)$, followed by sub clinical hypothyroidism (15\%), hyperthyroidism and Hashimoto thyroiditis, each consisting of $3.3 \%$ and the least was cancer, $1.7 \%$. At least 13 million Americans suffer from thyroid disorders, and in more than $80 \%$ of cases, the problem is an underactive thyroid gland - hypothyroidism. 7Likewise, other study have showed that hypothyroidism is such a common problem with an incidence of 410/100000/yearin women and $60 / 100000 /$ year in men, increasing with age). ${ }^{1}$ In the general population,the prevalence of subclinical hypothyroidism is about 4 to 8.5 percent, and may be as high as up to 20 percent in women older than 60 years. ${ }^{8}$, 2Other thyroid disorders are comparatively less in incidence.

Evaluation of psychiatric co-morbidity among the subjects with thyroid disorder showed that; greater numbers of the subjects i.e. $48.3 \%$ (29) had neurotic, stress- related and somatoform disorders (F40- F48) followed by $26.7 \%$ (16) of mood (affective) disorder (F30- F39). Others comorbidities were headache and lack or loss of sexual desire. Few had two simultaneously occurring psychiatric disorders.

Among the neurotic, stress- related and somatoform disorders i.e. (F40- F48) category; anxiety disorder unspecified $=17$; somatization disorder $=5$; mixed anxiety disorder $=2$; panic disorder $=2$. There were patients a single diagnosis of obsessive- compulsive disorder, persistent somatoform pain disorder and neuroasthenia.

Psychiatric presentations are often the first sign of thyroid disorders. Anxiety and progressive mental slowing associated with diminished recent memory, speech deficits and diminished learning ability are the characteristic initial progression of symptoms that occur in hypothyroidism. Rogers et al examined the prevalence and characteristics of medical illness in 711 patients who were enrolled in the Harvard-Brown Anxiety Disorders Research Program (HARP), a multi-center, longitudinal study of anxiety disorders. The study noted that patients who suffered from panic disorder were more likely to have an underlying medical illness causing their anxiety, particularly thyroid disease in women. ${ }^{6}$

The vast majority of patients with hyperthyroidism will display a psychiatric 
disorder such as anxiety, mania, or depression. ${ }^{3}$ Between $30 \%$ and $40 \%$ present with conspicuous complaints of anxiety, nervousness, apprehension, dread, depression, restlessness, diminished concentration, forced thinking, emotional lability, and hyperkinesia. 6 The occurrence of anxiety as a symptom of hyperthyroidism is well recognized. In one study, 29 patients were prospectively followed and found that 23 of them were diagnosed with generalized anxiety disorder and/or panic disorder. ${ }^{2}$ Similarly, Trepacz et al report a highprevalence of general anxiety disorder in a series of patients with untreated Graves' disease. In a study of patients with Hashimoto's thyroiditis, anxiety was a prominent initial symptom at the time the condition was diagnosed. Popkin and MacKenzie note that the behavioral changes of hyperthyroidism are numerous and useful in differentiating it from a primary anxiety neurosis or a neurasthenia. Patients with hyperthyroidism are differentiated from primary anxiety states as "in thyroid dysfunction, sleeping pulse will remain accelerated; sedated pulse will exceed 80; palms will be dry and warm, not cold and clammy; fatigue will be accomplished by a desire to be active; and cognitive dysfunction is more prominent than in neurasthenia." 6

Whybrow et al noted the elevation of schizophrenia and paranoid scales on the MMPI when patients are hyperthyroid and psychotic. MacCrimmon et al noted MMPI changes in hyperthyroid patients suggestive of hysterical somatization. They suggested that the behavioral, neurotic and psychotic manifestations of hyperthyroidism were related more to disease induced biochemical abnormalities than to the patient's previous personality pattern. ${ }^{6}$ Psychiatric symptoms of anxiety in hyperthyroidism appears to be mediated by beta-adrenergic hyperactivity. ${ }^{4}$, 3

Among the mood (affective) disorder i.e. (F30 F39) category; recurrent depressive disorder $=6$; bipolar affective disorder $=4$, depressive episode=3; dysthymia $=2$ and mania $=1$.

Depression is usually linked to hypothyroidism. ${ }^{3}$ The lifetime frequency of depression was significantly higher in the subjects who met the criteria for subclinical hypothyroidism (56\%) than in those who did not $(20 \%)$, suggesting that subclinical hypothyroidism may lower the threshold for the occurrence of depression. ${ }^{9}$ Only a few instances of mania or hypomania associated with hypothyroidism have been reported in the literature. Underlying mechanisms are less clear; they could include dysregulation of CNS catecholamine receptor sensitivity, associated thyroditis and thyrotoxicosis, or a disruption of circadian rhythms. ${ }^{4}$ The relationship between hyperthyroidism and depression is less clear. However, prolonged hyperthyroidism might exhaust noradrenergic transmission and thus contribute to depression. Noradrenergic exhaustion might well occur in patients with hyperthyroidism who have bipolar disorder. In the initial phase of hyperthyroidism, thyroid hormone stimulation of the noradrenergic system may cause mania; later, when noradrenergic neurotransmission is exhausted, it may contribute to depression. ${ }^{3}$ Accumulating evidence suggests that hypothalamo-pituitary-thyroid (HPT) axis dysfunction is relevant to the pathophysiology and clinical course of bipolar affective disorder. Hypothyroidism, either overt or more commonly subclinical, appears to the commonest abnormality found in bipolar disorder. The prevalence of thyroid dysfunction is also likely to be greater among patients with rapid cycling and other refractory forms of the disorder. 10

Even though thyroid disorders are associated with psychiatric symptoms in clinical populations, existence of a similar association in general population is less certain. ${ }^{4}$ Data from epidemiological studies provide conflicting evidence as to associations between thyroid disorders and mental symptoms. ${ }^{3}$

In one such survey, Canadian researchers found that the only psychiatric disorder associated with thyroid disease was social anxiety disorder (social phobia). In a study of more than 300 people over age 60 who came to internal medicine and psychiatry clinics, some of them for depression and others for symptoms suggesting abnormal thyroid activity, researchers found a high rate of depression among those with subclinical 
hypothyroidism but not those with clinical hypothyroidism. So the influence of thyroid deficiency on mental health remains uncertain. But the findings may conflict because studies have selected patients and evaluated depressive symptoms and thyroid function by different standards. ${ }^{7}$

But the other study showed higher rates of panic disorder, simple phobia, obsessive-compulsive disorder, major depressive disorder, bipolar disorder and cyclothymia in thyroid patients than in the general population. These findings would suggest that the co-occurrence of psychiatric and thyroid diseases may be the result of common biochemical abnormalities. ${ }^{5}$

\section{CONCLUSION}

Females are more likely to have thyroid disorder. As age increases, incidences of thyroid disorder also increase. Clinician has to be alert to the possible underlying psychiatric disorder in subjects with any thyroid disorder.

\section{REFERNECES}

1. Mistry N, Wass J, Turner MR. When to consider thyroid dysfunction in the neurology clinic. PractNeurol 2009; 9: 145-56.
2. The role of your thyroid in psychiatric illness, Charles Meusburger, Article Source: http://EzineArticles.com/5506756

3. Robertas B, Arthur J, Prange Jr. Thyroid Disease and Mental Disorders: Cause and Effect or Only co morbidity?CurrOpin Psychiatry 2010; 23(4): 36668.

4. Chakrabarti S. Thyroid Functions and Bipolar Affective Disorder. Journal of Thyroid Research, vol. 2011, Article ID 306367, 13 pages, 2011. doi:10.4061/2011/306367

5. Placidi G.P.A, Boldrini M, Patronelli A et al. Prevalence of Psychiatric Disorders in Thyroid Diseased Patients. Neuropsychobiology 1998; 38: 222-5.

6. Hall R.C.W, Hall R.C.W. Anxiety and Endocrine Disease. Seminars in Clinical Neuropsychiatry. (ed Michael K. Popkin). John Hopkins University.

7. Patten SB, et al. Self-Reported Thyroid Disease and Mental Disorder Prevalence in the General Population. General Hospital Psychiatry 2006; 28(6): 503-08.

8. Wilson GR, Curry RW, Jr. Subclinical thyroid disease. Am Fam Physician. 2005; 15; 72(8): 151724.

9. Haggerty JJ Jr, Stern RA, Mason GA et al. Subclinical hypothyroidism: a modifiable risk factor for depression? Am J Psychiatry 1993; 150(3): 508-10.

10. Chakrabarti S. Thyroid functions and bipolar affective disorder. Journal of Thyroid Research 2011; 2011:306367. 